\title{
BUILDING SOME SYMMETRIC LAGUERRE-HAHN \\ FUNCTIONALS OF CLASS TWO AT MOST THROUGH \\ THE SUM OF SYMMETRIC FUNCTIONALS AS \\ PSEUDOFUNCTIONS WITH A DIRAC MEASURE AT ORIGIN
}

\author{
M. SGHAIER AND J. ALAYA \\ Received 16 May 2005; Revised 4 March 2006; Accepted 4 May 2006
}

We show that if $v$ is a symmetric regular Laguerre-Hahn linear form (functional), then the linear form $u$ defined by $u=-\lambda x^{-2} v+\delta_{0}$ is also regular and symmetric LaguerreHahn linear form for every complex $\lambda$ except for a discrete set of numbers depending on $v$. We explicitly give the coefficients of the second-order recurrence relation, the structure relation of the orthogonal sequence associated with $u$, and the class of the linear form $u$ knowing that of $v$. Finally, we apply the above results to the symmetric associated form of the first order for the classical polynomials.

Copyright ( 2006 Hindawi Publishing Corporation. All rights reserved.

\section{Introduction}

The product of a linear form by a polynomial is one of the construction processes of linear forms. Christoffel has proved that the product of a positive definite linear form by a positive polynomial gives a positive definite linear form [7]. This result has been generalized in [9]. It was proved that, on certain regularity conditions, the product of a regular linear form $u$ by a polynomial $R$ gives a regular linear form. In particular, if $u$ is a semiclassical [14] (resp., Laguerre-Hahn $[2,8]$ ), then the linear form $R u$, if it is regular, is also semiclassical (resp., Laguerre-Hahn). Then it is interesting to consider the inverse problem, which consists in determining all regular linear forms $u$, satisfying $R u=-\lambda v$, where $v$ is a given regular linear form and $\lambda \neq 0$. When $R(x)=x-c, x^{2}$ (resp., $R(x)=x^{3}$ ), Maroni $[13,15]$ (resp., Maroni and Nicolau [16]) found necessary and sufficient conditions for $u$ to be regular. Also, an explicit expression for the orthogonal polynomials (OP) with respect to $u$ is proved. Finally, it was proved that, if $v$ is semiclassical linear form (see $[1,3,13,16])$, then $u$ is a semiclassical linear form. See also [11]. In particular, in this paper, Marcellán and Prianes proved that if $v$ is Laguerre-Hahn linear form, then $u$ is also a Laguerre-Hahn linear form. When $R(x)$ is of degree two, Branquinho and Marcellán [4] found necessary and sufficient conditions for $u$ to be regular. More generally, when $R(x)$ is any nonzero polynomial, Lee and Kwon [10] found a necessary and sufficient condition for $u$ to be regular and gave its corresponding OP in terms of the OP relative to $v$.

Hindawi Publishing Corporation

International Journal of Mathematics and Mathematical Sciences

Volume 2006, Article ID 70835, Pages 1-19

DOI 10.1155/IJMMS/2006/70835 
In this paper, we consider the same problem as in $[1,3]$ in the symmetric LaguerreHahn case: given a symmetric Laguerre-Hahn linear form $v$, find the linear form $u$ defined by $u=-\lambda x^{-2} v+\delta \Leftrightarrow x^{2} u=-\lambda v,(u)_{1}=0$. Section 2 is devoted to the preliminary results and notations used in the sequel. In Section 3, an explicit necessary and sufficient condition for the regularity of the new linear form different from that in [15] is given. We compute the exact class of the Laguerre-Hahn linear form obtained by the above modification and we give the structure relation of the OP sequence relatively to the linear form $u$. Finally, we apply our results to some examples.

\section{Notations and preliminary results}

Let $\mathscr{P}$ be the vector space of polynomials with coefficients in $\mathbb{C}$ and let $\mathscr{P}^{\prime}$ be its dual. We denote by $\langle u, f\rangle$ the action of $u \in \mathscr{P}^{\prime}$ on $f \in \mathscr{P}$. In particular, we denote by $(u)_{n}:=$ $\left\langle u, x^{n}\right\rangle, n \geq 0$, the moments of $u$. For any linear form $u$ and any polynomial $h$, we let $D u=$ $u^{\prime}, h u, \delta$ and $x^{-1} u$ be the linear forms defined by $\left\langle u^{\prime}, f\right\rangle:=-\left\langle u, f^{\prime}\right\rangle,\langle h u, f\rangle:=\langle u, h f\rangle$, $\langle\delta, f\rangle:=f(0)$, and $\left\langle x^{-1} u, f\right\rangle:=\left\langle u, \theta_{0} f\right\rangle$ where $\left(\theta_{0} f\right)(x)=(f(x)-f(0)) / x, f \in \mathscr{P}$.

Then, it is straightforward to prove that for $f \in \mathscr{P}$ and for $u \in \mathscr{P}^{\prime}$, we have

$$
\begin{gathered}
x\left(x^{-1} u\right)=u, \\
x^{-1}(x u)=u-(u)_{0} \delta .
\end{gathered}
$$

We also define the right multiplication of a linear form by a polynomial with

$$
(u h)(x):=\left\langle u, \frac{x h(x)-\xi h(\xi)}{x-\xi}\right\rangle=\sum_{m=0}^{n}\left(\sum_{j=m}^{n} a_{j}(u)_{j-m}\right) x^{m}, \quad h(x)=\sum_{j=0}^{n} a_{j} x^{j} .
$$

Next, it is possible to define the product of two linear forms through

$$
\langle u v, f\rangle:=\langle u, v f\rangle, \quad f \in \mathscr{P} .
$$

For $f, g \in \mathscr{P}$ and $u, v \in \mathscr{P}^{\prime}$, we have the following results [14]:

$$
\begin{gathered}
\left(u \theta_{0} f\right)(x)=\left(\theta_{0}(u f)\right)(x), \\
f(u v)=(f u) v+x\left(u \theta_{0} f\right)(x) v, \\
(f u)^{\prime}=f u^{\prime}+f^{\prime} u .
\end{gathered}
$$

Let us define the operator $\sigma: \mathscr{P} \longrightarrow \mathscr{P}$ by $(\sigma f)(x):=f\left(x^{2}\right)$. Then, we define the even part $\sigma u$ of $u$ by $\langle\sigma u, f\rangle:=\langle u, \sigma f\rangle$.

Therefore, we have [12]

$$
\begin{gathered}
f(x)(\sigma u)=\sigma\left(f\left(x^{2}\right) u\right), \\
\sigma u^{\prime}=2(\sigma(x u))^{\prime} .
\end{gathered}
$$

The linear form $v$ will be called regular if we can associate with it a sequence $\left\{S_{n}\right\}_{n \geq 0}$ $\left(\operatorname{deg}\left(S_{n}\right) \leq n\right)$ such that

$$
\left\langle v, S_{n} S_{m}\right\rangle=r_{n} \delta_{n m}, \quad n, m \geq 0, r_{n} \neq 0, n \geq 0 .
$$


Then, $\operatorname{deg}\left(S_{n}\right)=n, n \geq 0$ and we can always suppose each $S_{n}$ a monic. The sequence $\left\{S_{n}\right\}_{n \geq 0}$ is said to be orthogonal with respect to $v$.

It is a very well-known fact that the sequence $\left\{S_{n}\right\}_{n \geq 0}$ satisfies the recurrence relation (see, e.g., the monograph by Chihara [6])

$$
\begin{gathered}
S_{n+2}(x)=\left(x-\xi_{n+1}\right) S_{n+1}(x)-\delta_{n+1} S_{n}(x), \quad n \geq 0, \\
S_{1}(x)=x-\xi_{0}, \quad S_{0}(x)=1
\end{gathered}
$$

with $\left(\xi_{n}, \delta_{n+1}\right) \in \mathbb{C} \times(\mathbb{C}-\{0\}), n \geq 0$, by convention we set $\delta_{0}=(v)_{0}=1$.

In this case, we let $\left\{S_{n}^{(1)}\right\}_{n \geq 0}$ be the associated sequence of first order for the sequence $\left\{S_{n}\right\}_{n \geq 0}$ satisfying the second order recurrence relation

$$
\begin{gathered}
S_{n+2}^{(1)}(x)=\left(x-\xi_{n+2}\right) S_{n+1}^{(1)}(x)-\delta_{n+2} S_{n}^{(1)}(x), \quad n \geq 0, \\
S_{1}^{(1)}(x)=x-\xi_{1}, \quad S_{0}^{(1)}(x)=1, \quad\left(S_{-1}^{(1)}(x)=0\right) .
\end{gathered}
$$

Another important representation of $S_{n}^{(1)}(x)$ is, (see [5]),

$$
S_{n}^{(1)}(x):=\left\langle v, \frac{S_{n+1}(x)-S_{n+1}(\zeta)}{x-\zeta}\right\rangle
$$

Also, let $\left\{S_{n}(\cdot, \mu)\right\}_{n \geq 0}$ be the corecursive polynomials for the sequence $\left\{S_{n}\right\}_{n \geq 0}$ satisfying [5]

$$
S_{n}(x, \mu)=S_{n}(x)-\mu S_{n-1}^{(1)}(x), \quad n \geq 0 .
$$

A linear form $v$ is called symmetric if $(v)_{2 n+1}=0, n \geq 0$. In (2.9), we have $\xi_{n}=0, n \geq 0$ [6].

Throughout this paper, unless otherwise mentioned, the linear form $v$ will be supposed normalized, (i.e., $(v)_{0}=1$ ), symmetric, and regular.

Let us consider the quadratic decomposition of $\left\{S_{n}\right\}_{n \geq 0}$ and $\left\{S_{n}^{(1)}\right\}_{n \geq 0}[6,12]$ :

$$
\begin{gathered}
S_{2 n}(x)=\widetilde{P}_{n}\left(x^{2}\right), \quad S_{2 n+1}(x)=x \widetilde{R}_{n}\left(x^{2}\right), \\
S_{2 n}^{(1)}(x)=\widetilde{R}_{n}\left(x^{2},-\delta_{1}\right), \quad S_{2 n+1}^{(1)}(x)=x \widetilde{P}_{n}^{(1)}\left(x^{2}\right) .
\end{gathered}
$$

The sequences $\left\{\widetilde{P}_{n}\right\}_{n \geq 0}$ and $\left\{\widetilde{R}_{n}\right\}_{n \geq 0}$ are, respectively, orthogonal with respect to $\sigma v$ and $x \sigma v$. We also have

$$
\begin{gathered}
\widetilde{P}_{n+2}(x)=\left(x-\xi_{n+1}^{\widetilde{P}}\right) \widetilde{P}_{n+1}(x)-\delta_{n+1}^{\widetilde{P}} \widetilde{P}_{n}(x), \quad n \geq 0, \\
\widetilde{P}_{1}(x)=x-\xi_{0}, \quad \widetilde{P}_{0}(x)=1,
\end{gathered}
$$

with

$$
\xi_{0}^{\widetilde{P}}=\delta_{1}, \quad \xi_{n+1}^{\widetilde{P}}=\delta_{2 n+2}+\delta_{2 n+3}, \quad \delta_{n+1}^{\widetilde{P}}=\delta_{2 n+1} \delta_{2 n+2}, \quad n \geq 0
$$


4 Symmetric Laguerre-Hahn linear forms

By virtue of (2.9), with $\xi_{n}=0$, we get $S_{n+2}(0)=-\delta_{n+1} S_{n}(0)$. Consequently,

$$
S_{0}(0)=\widetilde{P}_{0}(0)=1, \quad S_{2 n+2}(0)=\widetilde{P}_{n+1}(0)=(-1)^{n+1} \prod_{v=0}^{n} \delta_{2 v+1}, \quad n \geq 0 .
$$

Proposition 2.1 [14]. $v$ is regular if and only if $\sigma v$ and $x \sigma v$ are regular.

\section{The Laguerre-Hahn case}

For a $\lambda \in \mathbb{C}-\{0\}$, we can define a new linear form in $\mathscr{P}^{\prime}$,

$$
u=-\lambda x^{-2} v+\delta_{0}
$$

From (2.1) and (3.1), we have

$$
x^{2} u=-\lambda v
$$

Remark 3.1. The above problem was partially treated by the second author and Maroni $[1,15]$ and we are going to handle it differently.

Proposition 3.2. $u$ is regular if and only if $\widetilde{P}_{n}(0, \lambda) \neq 0, n \geq 0$, where $\widetilde{P}_{n}$ is defined by (2.13).

Proof. Applying the operator $\sigma$ for (3.2) and using (2.6), we obtain

$$
x \sigma u=-\lambda \sigma v .
$$

From (2.2) and (3.3), we get

$$
\sigma u=-\lambda x^{-1} \sigma v+\delta_{0}
$$

From (3.1), it is plain that $u$ is symmetric linear form. Then, according to Proposition $2.1 u$ is regular if and only if $x \sigma u$ and $\sigma u$ are regular. But $x \sigma u=-\lambda \sigma v$ is regular because $\lambda \neq 0$ and $\sigma v$ is regular. So $u$ is regular if and only if $\sigma u=-\lambda x^{-1} \sigma v+\delta_{0}$ is regular.

Or, $\left\{\widetilde{P}_{n}\right\}_{n \geq 0}$ is the corresponding orthogonal sequence to $\sigma v$, and it was shown in [13] that $\sigma u=-\lambda x^{-1} \sigma v+\delta_{0}$ is regular if and only if $\lambda \neq 0$ and $\widetilde{P}_{n}(0, \lambda) \neq 0, n \geq 0$. Then, we deduce the desired result.

Remarks 3.3. (1) $u$ is regular if and only if $\lambda \neq \lambda_{n}, n \geq 0$, where $\lambda_{n}=\widetilde{P}_{n}(0) / \widetilde{P}_{n-1}^{(1)}(0)$.

(2) In fact, we have the well-known identity (see [6, page 86$]$ )

$$
\widetilde{P}_{n+1}(0) \widetilde{P}_{n+1}^{(1)}(0)-\widetilde{P}_{n+2}(0) \widetilde{P}_{n}^{(1)}(0)=\prod_{v=0}^{n} \delta_{v+1}^{\widetilde{P}}, \quad n \geq 0 .
$$

Dividing the above equation by $\widetilde{P}_{n+2}(0) \widetilde{P}_{n+1}(0)$ and using (2.13), (2.16), and (2.17), we obtain

$$
\frac{\widetilde{P}_{n+1}^{(1)}(0)}{\widetilde{P}_{n+2}(0)}-\frac{\widetilde{P}_{n}^{(1)}(0)}{\widetilde{P}_{n+1}(0)}=-\prod_{v=0}^{n+1} \frac{\delta_{2 v}}{\delta_{2 v+1}}, \quad n \geq 0
$$


This leads to

$$
\widetilde{P}_{n}^{(1)}(0)=-\widetilde{P}_{n+1}(0) \sum_{k=0}^{n} \prod_{v=0}^{k} \frac{\delta_{2 v}}{\delta_{2 v+1}}, \quad n \geq 0 .
$$

Using (2.12) and (3.7), we can easily find the result given in [15] according to Proposition 3.2: when the linear form $v$ is symmetric, then

$$
u \text { is regular } \Longleftrightarrow \lambda \neq-\left(\sum_{k=0}^{n} \prod_{v=0}^{k} \frac{\delta_{2 v}}{\delta_{2 v+1}}\right)^{-1} .
$$

When $u$ is regular, let $\left\{Z_{n}\right\}_{n \geq 0}$ be the corresponding sequence satisfying the recurrence relation

$$
\begin{gathered}
Z_{n+2}(x)=x Z_{n+1}(x)-\gamma_{n+1} Z_{n}(x), \quad n \geq 0, \\
Z_{1}(x)=x, \quad Z_{0}(x)=1 .
\end{gathered}
$$

Let us now consider the quadratic decomposition of the sequence $\left\{Z_{n}\right\}_{n \geq 0}$,

$$
Z_{2 n}(x)=P_{n}\left(x^{2}\right), \quad Z_{2 n+1}(x)=x R_{n}\left(x^{2}\right), \quad n \geq 0 .
$$

From (3.4), we can deduce the following result.

Proposition 3.4 [13]. The polynomials of the sequence $\left\{P_{n}\right\}_{n \geq 0}$ satisfy the relation

$$
P_{n+1}(x)=\tilde{P}_{n+1}(x)+\tilde{a}_{n} \widetilde{P}_{n}(x), \quad n \geq 0,
$$

where

$$
\tilde{a}_{n}=-\frac{\widetilde{P}_{n+1}(0, \lambda)}{\widetilde{P}_{n}(0, \lambda)}, \quad n \geq 0 .
$$

LEMMA 3.5 (cf. [1]).

$$
Z_{n+2}(x)=S_{n+2}(x)+a_{n} S_{n}(x), \quad n \geq 0,
$$

with

$$
a_{2 n}=\tilde{a}_{n}, \quad a_{2 n+1}=\delta_{2 n+2}, \quad n \geq 0 .
$$

Proof. On the basis of [1], we have $a_{2 n+1}=\delta_{2 n+2}, n \geq 0$ and

$$
Z_{2 n+2}(x)=S_{2 n+2}(x)+a_{2 n} S_{2 n}(x), \quad n \geq 0 .
$$

In (3.13), replace $x$ by $x^{2}$ and compare the obtained equation with the above one, we obtain $a_{2 n}=\tilde{a}_{n}, n \geq 0$, according to (3.10).

Proposition 3.6 (cf. [15]).

$$
\gamma_{1}=-\lambda, \quad \gamma_{2 n+2}=\tilde{a}_{n}, \quad \gamma_{2 n+3}=\frac{\delta_{2 n+1} \delta_{2 n+2}}{\tilde{a}_{n}}, \quad n \geq 0 .
$$


Remarks 3.7. (1) The decomposition (3.10) shows that $R_{n}(x)=\widetilde{P}_{n}(x), n \geq 0$, since $x \sigma u=$ $-\lambda \sigma v$. Consequently, the polynomial $Z_{2 n+1}$ does not depend on $\lambda$.

(2) From (2.16), (3.9), and (3.16), the sequence $\left\{P_{n}\right\}_{n \geq 0}$ satisfies the recurrence relation (2.13) with

$$
\begin{array}{ccc}
\beta_{0}^{P}=-\lambda, \quad \beta_{n+1}^{P}=\tilde{a}_{n}+\frac{\delta_{2 n+1} \delta_{2 n+2}}{\tilde{a}_{n}}, & n \geq 0, \\
\gamma_{1}^{P}=-\lambda \tilde{a}_{0}, & \gamma_{n+2}^{P}=\frac{\tilde{a}_{n+1} \delta_{2 n+1} \delta_{2 n+2}}{\tilde{a}_{n}}, & n \geq 0 .
\end{array}
$$

Definition 3.8 [2]. The regular linear form $v$ is called Laguerre-Hahn if its formal Stieltjes function satisfies the Riccati equation

$$
\widetilde{\Phi}(z) S^{\prime}(v)(z)=\widetilde{B}(z) S^{2}(v)(z)+\widetilde{C}_{0}(z) S(v)(z)+\widetilde{D}_{0}(z),
$$

where $\widetilde{\Phi}$ is monic, $\widetilde{B}, \widetilde{C}_{0}$, and $\widetilde{D}_{0}$ are polynomials, and $S(v)(z)=-\sum_{n \geq 0}\left((v)_{n} / z^{n+1}\right)$.

It was shown in [8] that $(3.18)$ is equivalent to

$$
(\tilde{\Phi}(x) v)^{\prime}+\tilde{\Psi} v+\widetilde{B}\left(x^{-1} v^{2}\right)=0
$$

with

$$
\tilde{\Psi}(x)=-\tilde{\Phi}^{\prime}(x)-\widetilde{C}_{0}(x)
$$

We also have the following relation:

$$
\widetilde{D}_{0}(x)=-\left(v \theta_{0} \tilde{\Phi}\right)^{\prime}(x)-\left(v \theta_{0} \tilde{\Psi}\right)(x)-\left(v^{2} \theta_{0}^{2} \widetilde{B}\right)(x) .
$$

Remark 3.9 [14]. When $B=0$ in (3.18) or (3.19), the linear form $v$ is semiclassical.

Proposition 3.10 [1]. Define $\tilde{d}=\max (\operatorname{deg}(\tilde{\Phi}), \operatorname{deg}(\widetilde{B}))$ and $\tilde{p}=\operatorname{deg}(\tilde{\Psi})$.

The Laguerre-Hahn linear form $v$ satisfying (3.19) is of class $\tilde{s}=\max (\tilde{d}-2, \tilde{p}-1)$ if and only if

$$
\prod_{c \in \mathscr{L}}\left\{\left|\tilde{\Phi}^{\prime}(c)+\tilde{\Psi}(c)\right|+|\tilde{B}(c)|+\left|\left\langle v, \theta_{c}^{2} \tilde{\Phi}+\theta_{c} \tilde{\Psi}+v \theta_{0} \theta_{c} \tilde{B}\right\rangle\right|\right\} \neq 0
$$

where $\mathscr{Z}$ denotes the set of zeros of $\tilde{\Phi}$.

COROLlary 3.11. The Laguerre-Hahn linear form $v$ satisfying (3.18) is of class $\tilde{s}=\max (\tilde{d}-$ $2, \tilde{p}-1)$ if and only if

$$
\prod_{c \in \mathscr{F}}\left\{\left|\widetilde{C}_{0}(c)\right|+|\widetilde{B}(c)|+\left|\widetilde{D}_{0}(c)\right|\right\} \neq 0
$$


Remark 3.12. Equation (3.23) is equivalent to the fact that the polynomial coefficients in (3.18) are coprime.

Proposition 3.13. If $v$ is a Laguerre-Hahn linear form and satisfies (3.18), then for every $\lambda \in \mathbb{C}-\{0\}$ such that $\widetilde{P}_{n}(0, \lambda) \neq 0, n \geq 0$, the linear form $u$ defined by (3.1) is regular and Laguerre-Hahn. It satisfies

$$
\Phi(z) S^{\prime}(u)(z)=B(z) S^{2}(u)(z)+C_{0}(z) S(u)(z)+D_{0}(z),
$$

where

$$
\begin{gathered}
\Phi(z)=z^{2} \widetilde{\Phi}(z), \\
B(z)=-\lambda^{-1} z^{4} \widetilde{B}(z), \\
C_{0}(z)=z^{2} \widetilde{C}_{0}(z)-2 \lambda^{-1} z^{3} \widetilde{B}(z)-2 z \widetilde{\Phi}(z), \\
D_{0}(z)=-\widetilde{\Phi}(z)-\lambda \widetilde{D}_{0}(z)+z \widetilde{C}_{0}(z)-\lambda^{-1} z^{2} \widetilde{B}(z),
\end{gathered}
$$

and $u$ is of class such that $s \leq \tilde{s}+4$.

Proof. We have [14]

$$
\begin{aligned}
S(v)(z) & =S\left(-\lambda^{-1} \xi^{2} u\right)(z) \\
& =-\lambda^{-1}\left\{z^{2} S(u)(z)+\left(u \theta_{0}\left(\xi^{2}\right)\right)(z)\right\} \\
& =-\lambda^{-1}\left\{z^{2} S(u)(z)+z\right\}
\end{aligned}
$$

By substituting (3.26) in (3.18), we easily find (3.24)-(3.25).

The linear form $u$ satisfies the distributional equation

$$
(\Phi(x) u)^{\prime}+\Psi u+B\left(x^{-1} u^{2}\right)=0
$$

where $\Phi$ and $B$ are the polynomials defined by (3.25) and

$$
\Psi(x)=-\Phi^{\prime}(x)-C_{0}(x)=x^{2}\left(\tilde{\Psi}(x)+2 \lambda^{-1} x \widetilde{B}(x)\right) .
$$

Then $\operatorname{deg}(\Phi) \leq \tilde{s}+4, \operatorname{deg}(B) \leq \tilde{s}+6$, and $\operatorname{deg}(\Psi)=p \leq \tilde{s}+5$.

Thus $d=\max (\operatorname{deg}(\Phi), \operatorname{deg}(B)) \leq \tilde{s}+6$ and $s=\max (d-2, p-1) \leq \tilde{s}+4$.

Proposition 3.14. The class of $u$ depends only on the zero $x=0$ of $\Phi$.

Proof. Since $v$ is a Laguerre-Hahn linear form of class $s, S(v)(z)$ satisfies (3.18), where the polynomials $\widetilde{\Phi}, \widetilde{B}, \widetilde{C}_{0}$, and $\widetilde{D}_{0}$ are coprime. Let $\Phi, B, C_{0}$, and $D_{0}$ be as in Proposition 3.13. 
Let $c$ be a zero of $\Phi$ different from 0 , this implies that $\tilde{\Phi}(c)=0$. We know that $|\widetilde{B}(c)|+$ $\left|\widetilde{C}_{0}(c)\right|+\left|\tilde{D}_{0}(c)\right| \neq 0$,

(i) if $\widetilde{B}(c) \neq 0$, then $B(c) \neq 0$,

(ii) if $\widetilde{B}(c)=0$ and $\widetilde{C}_{0}(c) \neq 0$, then $C_{0}(c) \neq 0$,

(iii) if $\widetilde{B}(c)=\widetilde{C}_{0}(c)=0$, then $D_{0}(c) \neq 0$, whence $|B(c)|+\left|C_{0}(c)\right|+\left|D_{0}(c)\right| \neq 0$.

Concerning the class of $u$, we have the following result.

Proposition 3.15. Let $\tilde{t}=\operatorname{deg} \tilde{\Phi}, \tilde{r}=\operatorname{deg} \widetilde{B}$, and $\tilde{p}=\operatorname{deg} \tilde{\Psi}$. Under the conditions of Proposition 3.13, for the class of $u$, the following two different cases hold.

(1) If $-\widetilde{\Phi}(0)-\lambda \widetilde{D}_{0}(0) \neq 0$, then

$$
s= \begin{cases}\tilde{s}+4 & \text { if } p \tilde{<} \tilde{r}, \tilde{t} \leq \tilde{r} \\ \tilde{s}+3 & \text { if } \tilde{p}=\tilde{r}, \tilde{t}<\tilde{r}+2 \text { or } \tilde{p}<\tilde{r}, \tilde{t}=\tilde{r}+1 \\ \tilde{s}+2 & \text { if } \tilde{p} \leq r, \tilde{t} \geq \tilde{r}+2 \text { or } \tilde{p} \geq \tilde{r}+1\end{cases}
$$

(2) If $-\tilde{\Phi}(0)-\lambda \widetilde{D}_{0}(0)=0$ and $\Phi(0) \neq 0$, then

$$
s= \begin{cases}\tilde{s}+3 & \text { if } p \tilde{<} \tilde{r}, \tilde{t} \leq \tilde{r} \\ \tilde{s}+2 & \text { if } \tilde{p}=\tilde{r}, \tilde{t}<\tilde{r}+2 \text { or } \tilde{p}<\tilde{r}, \tilde{t}=\tilde{r}+1 \\ \tilde{s}+1 & \text { if } \tilde{p} \leq r, \tilde{t} \geq \tilde{r}+2 \text { or } \tilde{p} \geq \tilde{r}+1\end{cases}
$$

where the polynomials $\widetilde{\Phi}, \widetilde{\Psi}, \widetilde{B}, \widetilde{C}_{0}$, and $\widetilde{D}_{0}$ are defined in (3.18)-(3.19).

Proof. If $-\tilde{\Phi}(0)-\lambda \widetilde{D}_{0}(0) \neq 0$, then from (3.25) and (3.28) we have

$$
\operatorname{deg}(\Phi)=\tilde{t}+2, \quad \operatorname{deg}(B)=\tilde{r}+4, \quad p:=\operatorname{deg}(\Psi) \leq \max (\tilde{p}+2, \tilde{r}+3)
$$

We will distinguish three cases:

(a) $\tilde{p}<\tilde{r}$, then $p=\tilde{r}+3$ and $s=\max (\tilde{t}, \tilde{r}+2)$. If $\tilde{t} \leq \tilde{r}$, then $s=\tilde{r}+2=\tilde{s}+4$. If $\tilde{t}=\tilde{r}+$ 1 , then $s=\tilde{r}+2=\tilde{s}+3$, since $\tilde{s}=\tilde{r}-1$ in this case. If $\tilde{t} \geq \tilde{r}+2$, then $s=\tilde{t}=\tilde{s}+2$;

(b) $\tilde{p}=\tilde{r}$, then $p=\tilde{r}+3=\tilde{p}+3$ and $s=\max (\tilde{t}, \tilde{p}+2)$. If $\tilde{t}<\tilde{p}+2$, then $s=\tilde{p}+2=$ $\tilde{s}+3$. If $\tilde{t} \geq \tilde{p}+2$, then $s=\tilde{t}=\tilde{s}+2$

(c) $\tilde{p}>\tilde{r}$, then $p \leq \tilde{p}+2$ and $s=\max (\tilde{t}, \tilde{p}+1)$.

Since $\tilde{t}=\tilde{s}+2$ or $\tilde{p}+1=\tilde{s}+2$, then $s=\tilde{s}+2$.

Therefore, from the above situations, we deduce

$$
s= \begin{cases}\tilde{s}+4 & \text { if } p \tilde{<} \tilde{r}, \tilde{t} \leq \tilde{r} \\ \tilde{s}+3 & \text { if } \tilde{p}=\tilde{r}, \tilde{t}<\tilde{r}+2 \text { or } \tilde{p}<\tilde{r}, \tilde{t}=\tilde{r}+1 \\ \tilde{s}+2 & \text { if } \tilde{p} \leq r, \tilde{t} \geq \tilde{r}+2 \text { or } \tilde{p} \geq \tilde{r}+1\end{cases}
$$


If $-\tilde{\Phi}(0)-\lambda \tilde{D}(0)=0$, then (3.24)-(3.25) is divisible by $z$. Thus,

$$
\begin{aligned}
z \widetilde{\Phi}(z) S^{\prime}(u)(z)= & -\lambda^{-1} z^{3} \widetilde{B}(z) S^{2}(u)(z)+\left\{-2 \widetilde{\Phi}(z)-2 \lambda^{-1} z^{2} \widetilde{B}(z)+z \widetilde{C}_{0}(z)\right\} S(u)(z) \\
& +\left\{-\left(\theta_{0} \widetilde{\Phi}\right)(z)-\lambda^{-1} z \widetilde{B}(z)+\widetilde{C}_{0}(z)-\lambda\left(\theta_{0} \widetilde{D}\right)(z)\right\} .
\end{aligned}
$$

If $\tilde{\Phi}(0) \neq 0$, it is not possible to simplify, which means that the class of $u$ is

$$
s= \begin{cases}\tilde{s}+3 & \text { if } p \tilde{<} \tilde{r}, \tilde{t} \leq \tilde{r} \\ \tilde{s}+2 & \text { if } \tilde{p}=\tilde{r}, \tilde{t}<\tilde{r}+2 \text { or } \tilde{p}<\tilde{r}, \tilde{t}=\tilde{r}+1 \\ \tilde{s}+1 & \text { if } \tilde{p} \leq r, \tilde{t} \geq \tilde{r}+2 \text { or } \tilde{p} \geq \tilde{r}+1\end{cases}
$$

Note that the sequence of orthogonal polynomials (OPs) relatively to a Laguerre-Hahn linear form has a structure relation [2]. Then, if we consider that the linear form $v$ is Laguerre-Hahn, its OPs $\left\{S_{n}\right\}_{n \geq 0}$ fulfills the following structure relation:

$$
\tilde{\Phi}(x) S_{n+1}^{\prime}(x)-\widetilde{B}(x) S_{n}^{(1)}(x)=\frac{1}{2}\left(\widetilde{C}_{n+1}(x)-\widetilde{C}_{0}(x)\right) S_{n+1}(x)-\delta_{n+1} \widetilde{D}_{n+1}(x) S_{n}(x), \quad n \geq 0,
$$

with

$$
\begin{gathered}
\widetilde{C}_{n+1}(x)=-\widetilde{C}_{n}(x)+2 x \widetilde{D}_{n}(x), \\
\delta_{n+1} \widetilde{D}_{n+1}(x)=-\widetilde{\Phi}(x)+\delta_{n} \widetilde{D}_{n-1}(x)-x \widetilde{C}_{n}(x)+x^{2} \widetilde{D}_{n}(x),
\end{gathered}
$$

where $\widetilde{C}_{0}(x)$ and $\widetilde{D}_{0}(x)$ are given by $(3.18)$ and $\delta_{0} \widetilde{D}_{-1}(x)=\widetilde{B}(x)$.

Replacing $n$ by $n-1$ in (3.35) and using (2.9), we obtain

$$
\tilde{\Phi}(x) S_{n}^{\prime}(x)-\widetilde{B}(x) S_{n-1}^{(1)}(x)=\widetilde{D}_{n}(x) S_{n+1}(x)+\left(\frac{1}{2}\left(\widetilde{C}_{n}(x)-\widetilde{C}_{0}(x)\right)-x \widetilde{D}_{n}(x)\right) S_{n}(x) .
$$

According to Proposition 3.13, the linear form $u$ is also Laguerre-Hahn and its OPs $\left\{Z_{n}\right\}_{n \geq 0}$ satisfies a structure relation. In general, $\left\{Z_{n}\right\}_{n \geq 0}$ fulfills

$$
\Phi(x) Z_{n+1}^{\prime}(x)-B(x) Z_{n}^{(1)}(x)=\frac{1}{2}\left(C_{n+1}(x)-C_{0}(x)\right) Z_{n+1}(x)-\gamma_{n+1} D_{n+1}(x) Z_{n}(x), \quad n \geq 0,
$$

with

$$
\begin{gathered}
C_{n+1}(x)=-C_{n}(x)+2 x D_{n}(x), \\
\gamma_{n+1} D_{n+1}(x)=-\Phi(x)+\gamma_{n} D_{n-1}(x)-x C_{n}(x)+x^{2} D_{n}(x), \quad n \geq 0,
\end{gathered}
$$

where $C_{0}(x), D_{0}(x)$ are given by (3.25) and $\gamma_{0} D_{-1}(x)=B(x)$.

We are going to establish the expression of $C_{n}$ and $D_{n}, n \geq 0$, in terms of those of the sequence $\left\{S_{n}\right\}_{n \geq 0}$. 
Proposition 3.16. The sequence $\left\{Z_{n}\right\}_{n \geq 0}$ satisfies the structure relation (3.38) with (for $n \geq 0$ )

$$
\begin{gathered}
C_{n+2}(x)=x^{2} \widetilde{C}_{n+1}(x)+2\left(a_{n}-\delta_{n+1}\right) x \widetilde{D}_{n}(x)-2\left(a_{n+1}-\delta_{n+2}\right) x \widetilde{D}_{n+1}(x), \\
D_{n+2}(x)=x^{2} \widetilde{D}_{n+1}(x)+\left(a_{n}-\delta_{n+1}\right)\left(\widetilde{D}_{n}(x)-\frac{a_{n+1}}{a_{n}} \widetilde{D}_{n+2}(x)\right), \\
C_{1}=x^{2} \widetilde{C}_{0}(x)-2 \lambda x \widetilde{D}_{0}(x), \quad D_{1}(x)=x^{2} \widetilde{D}_{0}(x),
\end{gathered}
$$

$C_{0}(x)$ and $D_{0}(x)$ are given by (3.25).

To prove the above proposition, we need the following lemma.

LEMMA 3.17.

$$
\begin{array}{cc}
a_{n} x^{2} S_{n+1}(x)=a_{n} x Z_{n+2}+\gamma_{n+2}\left(\delta_{n+1}-a_{n}\right) Z_{n+1}(x), & n \geq 0, \\
a_{n} x^{2} S_{n}(x)=\left(a_{n+1}-\delta_{n+2}\right) Z_{n+2}+\gamma_{n+2} x Z_{n+1}(x), & n \geq 0, \\
x^{2} Z_{n+1}^{(1)}(x)=x Z_{n+2}(x)-\lambda\left(S_{n+1}^{(1)}(x)+a_{n} S_{n-1}^{(1)}(x)\right), & n \geq 0 .
\end{array}
$$

Proof. For (3.41)-(3.42) see [1].

It is clear that

$$
x\left(S_{n}(x)-S_{n}(\xi)\right)-(x-\xi)\left(S_{n}(x)-S_{n}(0)=\xi\left(S_{n}(x)-S_{n}(\xi)\right)+(\xi-x)\left(S_{n}(\xi)-S_{n}(0)\right),\right.
$$

then

$$
\left\langle\xi^{-2} v, \frac{S_{n}(\xi)-S_{n}(x)}{\xi-x}\right\rangle=\frac{S_{n-1}^{(1)}(x)-S_{n-1}^{(1)}(0)}{x^{2}}-\frac{S_{n-1}^{(1)^{\prime}}(0)}{x} .
$$

From (3.2) and (3.13), the conditions $\left\langle u, Z_{n+2}\right\rangle=0$ and $\left\langle u, x Z_{n+2}\right\rangle=0$ imply, respectively,

$$
\begin{gathered}
-\lambda\left(S_{n+1}^{(1)}{ }^{\prime}(0)+a_{n} S_{n-1}^{(1)}{ }^{\prime}(0)\right)+S_{n+2}(0)+a_{n} S_{n}(0)=0, \\
-\lambda\left(S_{n+1}^{(1)}(0)+a_{n} S_{n-1}^{(1)}(0)\right)=0 .
\end{gathered}
$$

From (2.11) and (3.13), we have

$$
\begin{aligned}
Z_{n+1}^{(1)}(x)= & \left\langle u, \frac{Z_{n+2}(\xi)-Z_{n+2}(x)}{\xi-x}\right\rangle \\
= & \frac{S_{n+2}(x)-S_{n+2}(0)}{x}+a_{n} \frac{S_{n}(x)-S_{n}(0)}{x} \\
& -\lambda\left\{\left\langle\xi^{-2} v, \frac{S_{n+2}(\xi)-S_{n+2}(x)}{\xi-x}\right\rangle+a_{n}\left\langle\xi^{-2} v, \frac{S_{n}(\xi)-S_{n}(x)}{\xi-x}\right\rangle\right\} .
\end{aligned}
$$

Taking into account (3.45)-(3.47) in the above equation, we get (3.43).

Now, we are able to prove Proposition 3.16. 
Proof. After derivation, we multiply (3.13) by $\tilde{\Phi}(x)$, we obtain

$$
\widetilde{\Phi}(x) Z_{n+2}^{\prime}(x)=\widetilde{\Phi}(x) S_{n+2}^{\prime}(x)+a_{n} \widetilde{\Phi}(x) S_{n}^{\prime}(x) .
$$

Multiplying (3.43) by $\lambda^{-1} \widetilde{B}(x)$, adding (3.49), and taking into account (3.35)-(3.37), we get

$$
\widetilde{\Phi}(x) Z_{n+2}^{\prime}(x)+\lambda^{-1} x^{2} \widetilde{B}(x) Z_{n+1}^{(1)}(x)=G(x, n) S_{n+1}(x)+H(x, n) S_{n}(x)+\lambda^{-1} x \widetilde{B}(x) Z_{n+2}(x),
$$

where

$$
\begin{aligned}
& G(x, n)=\frac{1}{2}\left(\widetilde{C}_{n+2}(x)-\widetilde{C}_{0}(x)\right) x+a_{n} \widetilde{D}_{n}(x)-\delta_{n+2} \widetilde{D}_{n+2}(x), \\
& H(x, n)=-\frac{1}{2}\left(\widetilde{C}_{n+2}(x)-\widetilde{C}_{0}(x)\right) \delta_{n+1}+a_{n}\left\{\frac{1}{2}\left(\widetilde{C}_{n}(x)-\widetilde{C}_{0}(x)\right)-x \widetilde{D}_{n}(x)\right\} .
\end{aligned}
$$

Multiplying (3.50) by $x^{2}$ and taking into account (3.14), (3.25), (3.36), and (3.41)(3.42), we obtain

$$
\begin{aligned}
\Phi(x) Z_{n+2}^{\prime}(x)-B(x) Z_{n+1}^{(1)}(x) \\
=\left\{\frac{1}{2} x^{2}\left(\widetilde{C}_{n+1}(x)-\widetilde{C}_{0}(x)\right)+\left(a_{n}-\delta_{n+1}\right) x \widetilde{D}_{n}(x)\right. \\
\left.\quad-\left(a_{n+1}-\delta_{n+2}\right) x \widetilde{D}_{n+1}(x)+x \widetilde{\Phi}(x)+\lambda^{-1} x^{3} \widetilde{B}(x)\right\} Z_{n+2}(x) \\
\quad-\gamma_{n+2}\left\{x^{2} \widetilde{D}_{n+1}(x)+\left(a_{n}-\delta_{n+1}\right)\left(\widetilde{D}_{n}(x)-\frac{a_{n+1}}{a_{n}} \widetilde{D}_{n+2}(x)\right)\right\} Z_{n+1}(x) .
\end{aligned}
$$

Comparing with (3.38), we get the desired result for $n \geq 2$.

Finally, using (3.39) with $n=0$ and (3.25), we easily obtain $C_{1}$ and $D_{1}$.

Remark 3.18. In the Laguerre-Hahn case, the polynomials $C_{n}$ and $D_{n}$ of (3.38) enable to obtain the coefficients of the fourth-order differential equation satisfied by each $Z_{n}$, $n \geq 0$. See, for instance [8, page 90$]$.

Examples 3.19. In the next examples, we apply our results to the associated linear form of the first order for the classical polynomials which are symmetric.

(1) Let $v$ be the associated linear form of the first order of Hermite. Here $[8,11]$

$$
\begin{gathered}
\delta_{2 n+1}=n+1, \quad \delta_{2 n+2}=\frac{2 n+3}{2}, \quad n \geq 0, \\
\tilde{\Phi}(x)=1, \quad \tilde{\Psi}(x)=2 x, \quad \tilde{B}(x)=-1, \\
\widetilde{C}_{n}(x)=-2 x, \quad \tilde{D}_{n}(x)=-2, \quad n \geq 0 .
\end{gathered}
$$

In this case, the linear form $v$ is a Laguerre-Hahn linear form of class $\widetilde{s}=0$. From (2.17) and (3.53), we have

$$
\widetilde{P}_{n}(0)=(-1)^{n} \Gamma(n+1), \quad n \geq 0 .
$$


From (3.53), (2.15), (2.16), and (2.10), we get the second-order recurrence relation satisfied by $\left\{\widetilde{P}_{n}^{(1)}\right\}_{n \geq 0}$. Using this relation, we deduce by induction

$$
\widetilde{P}_{n}^{(1)}(0)=2(-1)^{n+1}\left(\Gamma(n+2)-\frac{2 \Gamma(n+5 / 2)}{\sqrt{\pi}}\right), \quad n \geq 0
$$

From (2.12), and (3.55)-(3.56), we obtain

$$
\tilde{P}_{n}(0, \lambda)=\frac{(-1)^{n}}{\sqrt{\pi}}\left((1-2 \lambda) \sqrt{\pi} \Gamma(n+1)+4 \lambda \Gamma\left(n+\frac{3}{2}\right)\right), \quad n \geq 0,
$$

then $u$ is regular for every $\lambda \neq 0$ such that

$$
\lambda^{-1} \neq 2-\frac{4 \Gamma(n+3 / 2)}{\sqrt{\pi} \Gamma(n+1)}, \quad n \geq 0 .
$$

From (3.12) and (3.57), we obtain

$$
\tilde{a}_{n}=\frac{(1-2 \lambda) \sqrt{\pi} \Gamma(n+2)+4 \lambda \Gamma(n+5 / 2)}{(1-2 \lambda) \sqrt{\pi} \Gamma(n+1)+4 \lambda \Gamma(n+3 / 2)}, \quad n \geq 0 .
$$

Using (3.53), (3.59), and (3.16), we get

$$
\begin{aligned}
\gamma_{1} & =-\lambda, \\
\gamma_{2 n+2} & =\frac{(1-2 \lambda) \sqrt{\pi} \Gamma(n+2)+4 \lambda \Gamma(n+5 / 2)}{(1-2 \lambda) \sqrt{\pi} \Gamma(n+1)+4 \lambda \Gamma(n+3 / 2)}, \quad n \geq 0, \\
\gamma_{2 n+3} & =\frac{(1-2 \lambda) \sqrt{\pi}(2 n+3) \Gamma(n+2)+8 \lambda(n+1) \Gamma(n+5 / 2)}{2(1-2 \lambda) \sqrt{\pi} \Gamma(n+2)+8 \lambda \Gamma(n+5 / 2)}, \quad n \geq 0 .
\end{aligned}
$$

Taking into account that the linear form $v$ is Laguerre-Hahn and by virtue of Proposition 3.13, the linear form $u$ is also Laguerre-Hahn. It satisfies (3.24) and (3.27) with

$$
\begin{gathered}
\Phi(x)=x^{2}, \quad B(x)=\lambda^{-1} x^{4}, \quad \Psi(x)=\left(2-2 \lambda^{-1}\right) x^{3}, \\
C_{0}(x)=\left(2 \lambda^{-1}-2\right) x^{3}-2 x, \quad D_{0}(x)=\left(\lambda^{-1}-2\right) x^{2}+2 \lambda-1 .
\end{gathered}
$$

From (3.54), we have $-\widetilde{\Phi}(0)-\lambda \widetilde{D}_{0}(0)=-1+2 \lambda$ and $\Phi(0)=1$.

Now it is enough to use Proposition 3.15 to obtain the following.

(i) If $\lambda$ satisfies (3.58) and $\lambda^{-1} \neq 2$, then the class of $u$ is $s=2$.

(ii) If $\lambda^{-1}=2$, then the class of $u$ is $s=1$.

Remark 3.20. The symmetric Laguerre-Hahn linear forms of class $s=1$ have been described in [2]. 
Finally, from (3.54), (3.59), and Proposition 3.16, we give the elements of the structure relation of the sequence $\left\{Z_{n}\right\}_{n \geq 0}$ for $n \geq 0$,

$$
\begin{gathered}
C_{0}(x)=\left(2 \lambda^{-1}-2\right) x^{3}-2 x, \quad C_{1}(x)=-2 x^{3}+4 \lambda x, \\
C_{2 n+2}(x)=-2 x^{3}-\frac{6 \lambda \Gamma(n+3 / 2)}{(1-2 \lambda) \sqrt{\pi} \Gamma(n+1)+4 \lambda \Gamma(n+3 / 2)} x, \\
C_{2 n+3}(x)=-2 x^{3}+\frac{6 \lambda \Gamma(n+5 / 2)}{(1-2 \lambda) \sqrt{\pi} \Gamma(n+2)+4 \lambda \Gamma(n+5 / 2)} x, \\
D_{0}(x)=\left(\lambda^{-1}-2\right) x^{2}+2 \lambda-1, \quad D_{2 n+1}(x)=-2 x^{2}, \\
D_{2 n+2}(x)=-2 x^{2} \quad \lambda(2 \lambda-1) \sqrt{\pi} \Gamma(n+3 / 2) \Gamma(n+1) \\
-\frac{\lambda(n+2 \lambda) \sqrt{\pi} \Gamma(n+2)+4 \lambda \Gamma(n+5 / 2))}{2((1-2 \lambda) \sqrt{\pi} \Gamma(n+1)+4 \lambda \Gamma(n+3 / 2))((1-2 \lambda)} .
\end{gathered}
$$

(2) Let $v$ be the associated linear form of the first order of $\mathscr{f}(\alpha, \alpha)$. Here $[8,11]$

$$
\begin{gathered}
\delta_{2 n+1}=\frac{4(n+1)(n+\alpha+1)}{(4 n+2 \alpha+3)(4 n+2 \alpha+5)}, \\
\delta_{2 n+2}=\frac{(2 n+3)(2 n+2 \alpha+3)}{(4 n+2 \alpha+5)(4 n+2 \alpha+7)}, \quad n \geq 0, \\
\widetilde{\Phi}(x)=x^{2}-1, \quad \tilde{\Psi}(x)=-2(\alpha+2) x, \quad \widetilde{B}(x)=\frac{2 \alpha+1}{2 \alpha+3}, \\
\widetilde{C}_{n}(x)=2(n+\alpha+1) x, \quad \widetilde{D}_{n}(x)=2 n+2 \alpha+3, \quad n \geq 0 .
\end{gathered}
$$

We assume $(2 \alpha+1) \neq 0$, then $v$ is a Laguerre-Hahn linear form of class $\tilde{s}=0$.

By applying the same process as we did to obtain (3.57) and using the above results, we can get for $n \geq 0$,

$$
\widetilde{P}_{n}(0, \lambda)=\frac{(-1)^{n}}{\Gamma(2 n+\alpha+5 / 2)} d_{n}(\lambda), \quad n \geq 0,
$$

where

$$
\begin{aligned}
d_{n}(\lambda)= & (1-(2 \alpha+3) \lambda) \frac{\Gamma(\alpha+3 / 2) \Gamma(n+1) \Gamma(n+\alpha+1)}{\Gamma(\alpha+1)} \\
& +\frac{2(2 \alpha+3) \lambda}{\sqrt{\pi}} \Gamma\left(n+\frac{3}{2}\right) \Gamma\left(n+\alpha+\frac{3}{2}\right), \quad n \geq 0 .
\end{aligned}
$$

Then, $u$ is regular for every $\lambda \neq 0$ such that

$$
\lambda^{-1} \neq 2 \alpha+3-\frac{2 \Gamma(\alpha+1) \Gamma(n+3 / 2) \Gamma(n+\alpha+3 / 2)}{\sqrt{\pi} \Gamma(\alpha+3 / 2) \Gamma(n+1) \Gamma(n+\alpha+1)}, \quad n \geq 0 .
$$


14 Symmetric Laguerre-Hahn linear forms

From (3.12) and (3.65)-(3.66), we obtain

$$
\tilde{a}_{n}=\frac{4 d_{n+1}(\lambda)}{(4 n+2 \alpha+3)(4 n+2 \alpha+5) d_{n}(\lambda)}, \quad n \geq 0 .
$$

Using (3.63), (3.68), and (3.16), we get

$$
\begin{aligned}
\gamma_{1} & =-\lambda, \\
\gamma_{2 n+2} & =\frac{4 d_{n+1}(\lambda)}{(4 n+2 \alpha+3)(4 n+2 \alpha+5) d_{n}(\lambda)}, \quad n \geq 0, \\
\gamma_{2 n+3} & =\frac{(n+1)(n+\alpha+1)(2 n+3)(2 n+2 \alpha+3) d_{n}(\lambda)}{(4 n+2 \alpha+5)(4 n+2 \alpha+7) d_{n+1}(\lambda)}, \quad n \geq 0 .
\end{aligned}
$$

According to Proposition 3.13, the linear form $u$ is also Laguerre-Hahn. It satisfies (3.24) and (3.27) with

$$
\begin{gathered}
\Phi(x)=x^{2}\left(x^{2}-1\right), \quad B(x)=-\lambda^{-1} \frac{2 \alpha+1}{2 \alpha+3} x^{4}, \quad \Psi(x)=-2\left(\alpha+2-\lambda^{-1} \frac{2 \alpha+1}{2 \alpha+3}\right) x^{3}, \\
C_{0}(x)=2\left(\alpha-\lambda^{-1} \frac{2 \alpha+1}{2 \alpha+3}\right) x^{3}+2 x, \quad D_{0}(x)=\left(2 \alpha+1-\lambda^{-1} \frac{2 \alpha+1}{2 \alpha+3}\right) x^{2}+1-(2 \alpha+3) \lambda .
\end{gathered}
$$

From (3.64), we have $-\tilde{\Phi}(0)-\lambda \widetilde{D}_{0}(0)=1-\lambda(2 \alpha+3)$ and $\Phi(0)=-1$.

Now it is enough to use Proposition 3.15 to obtain the following.

(i) If $\lambda$ satisfies (3.67) and $\lambda^{-1} \neq 2 \alpha+3$, then the class of $u$ is $s=2$.

(ii) If $\lambda^{-1}=2 \alpha+3$, then the class of $u$ is $s=1$.

Finally, from Proposition 3.16, we have for $n \geq 0$,

$$
\begin{gathered}
C_{0}(x)=2\left(\alpha-\lambda^{-1} \frac{2 \alpha+1}{2 \alpha+3}\right) x^{3}+2 x, \quad C_{1}(x)=(2 \alpha+2) x^{3}-2 \lambda(2 \alpha+3) x, \\
C_{2 n+2}(x)=2(2 n+\alpha+2) x^{3}+\frac{4 \lambda(2 \alpha+3) \Gamma(n+3 / 2) \Gamma(n+\alpha+3 / 2)}{\sqrt{\pi} d_{n}(\lambda)} x, \\
C_{2 n+3}(x)=2(2 n+\alpha+3) x^{3}-\frac{4 \lambda(2 \alpha+3) \Gamma(n+5 / 2) \Gamma(n+\alpha+5 / 2)}{\sqrt{\pi} d_{n+1}(\lambda)} x, \\
D_{0}(x)=\left(2 \alpha+1-\lambda^{-1} \frac{2 \alpha+1}{2 \alpha+3}\right) x^{2}+1-(2 \alpha+3) \lambda, \\
D_{2 n+1}(x)=(4 n+2 \alpha+3) x^{2}, \\
=(4 n+2 \alpha+5) \quad \\
\times\left(x^{2}-\frac{(2 \alpha+3) \lambda(1-(2 \alpha+3) \lambda) \Gamma(\alpha+3 / 2) \Gamma(n+3 / 2) \Gamma(n+1) \Gamma(n+\alpha+1) \Gamma(n+\alpha+3 / 2)}{2 \sqrt{\pi} \Gamma(\alpha+1) d_{n}(\lambda) d_{n+1}(\lambda)}\right) .
\end{gathered}
$$


(3) Let $v$ be the associated linear form of the first order of $\mathscr{f}(\alpha,-\alpha)$. Here $[8,11]$

$$
\begin{gathered}
\delta_{2 n+1}=\frac{(2 n+\alpha+2)(2 n-\alpha+2)}{(4 n+3)(4 n+5)}, \\
\delta_{2 n+2}=\frac{(2 n+\alpha+3)(2 n-\alpha+3)}{(4 n+5)(4 n+7)}, \quad n \geq 0, \\
\widetilde{\Phi}(x)=x^{2}-1, \quad \tilde{\Psi}(x)=-4 x, \quad \widetilde{B}(x)=\frac{1-\alpha^{2}}{3}, \\
\widetilde{C}_{n}(x)=2(n+1) x, \quad \widetilde{D}_{n}(x)=2 n+3, \quad n \geq 0 .
\end{gathered}
$$

We assume $\left(1-\alpha^{2}\right) \neq 0$, then $v$ is a Laguerre-Hahn linear form of class $\widetilde{s}=0$.

From (2.17) and (3.72), we have

$$
\widetilde{P}_{n}(0)=(-1)^{n} \frac{\pi \sqrt{2} \Gamma(n+1+\alpha / 2) \Gamma(n+1-\alpha / 2)}{4^{n+1} \Gamma(n+9 / 4) \Gamma(n+7 / 4) \Gamma(1+\alpha / 2) \Gamma(1-\alpha / 2)}, \quad n \geq 0 .
$$

From (3.72), we have (with $\sum_{n=0}^{-1}=0$ )

$$
\begin{aligned}
\triangle_{n}= & \sum_{k=0}^{n} \prod_{v=0}^{k} \frac{\delta_{2 v}}{\delta_{2 v+1}}=\frac{15}{(2+\alpha)(2-\alpha)}+\frac{12 \Gamma(2+\alpha / 2) \Gamma(2-\alpha / 2)}{(2+\alpha)(2-\alpha) \Gamma(3 / 2+\alpha / 2) \Gamma(3 / 2-\alpha / 2)} \\
& \times \sum_{k=0}^{n-1} \frac{(4 k+9) \Gamma(k+5 / 2+\alpha / 2) \Gamma(k+5 / 2-\alpha / 2)}{4 \Gamma(k+3+\alpha / 2) \Gamma(k+3-\alpha / 2)}, \quad n \geq 0 .
\end{aligned}
$$

Or,

$$
\frac{(4 k+9) \Gamma(k+5 / 2+\alpha / 2) \Gamma(k+5 / 2-\alpha / 2)}{4 \Gamma(k+3+\alpha / 2) \Gamma(k+3-\alpha / 2)}=\tau_{k+1}-\tau_{k}
$$

with

$$
\tau_{k}=\frac{\Gamma(k+5 / 2+\alpha / 2) \Gamma(k+5 / 2-\alpha / 2)}{\Gamma(k+2+\alpha / 2) \Gamma(k+2-\alpha / 2)}, \quad k \geq 0,
$$

then

$$
\triangle_{n}=-3+3 \frac{\Gamma(1+\alpha / 2) \Gamma(1-\alpha / 2) \Gamma(n+5 / 2+\alpha / 2) \Gamma(n+5 / 2-\alpha / 2)}{\Gamma(3 / 2+\alpha / 2) \Gamma(3 / 2-\alpha / 2) \Gamma(n+2+\alpha / 2) \Gamma(n+2-\alpha / 2)}, \quad n \geq 0 .
$$

Therefore, from (3.7), (3.74), and the above result, we obtain

$$
\begin{gathered}
\widetilde{P}_{n}^{(1)}(0)=\frac{(-1)^{n+1} 3 \pi \sqrt{2}}{4^{n+1}}\left(\frac{\Gamma(n+2+\alpha / 2) \Gamma(n+2-\alpha / 2)}{\Gamma(n+9 / 4) \Gamma(n+7 / 4) \Gamma(1+\alpha / 2) \Gamma(1-\alpha / 2)}\right. \\
\left.-\frac{\Gamma(n+5 / 2+\alpha / 2) \Gamma(n+5 / 2-\alpha / 2)}{\Gamma(3 / 2+\alpha / 2) \Gamma(3 / 2-\alpha / 2)}\right) .
\end{gathered}
$$


From (2.12) and (3.74)-(3.79), we obtain

$$
\widetilde{P}_{n}(0, \lambda)=\frac{(-1)^{n} \pi \sqrt{2}}{4^{n+1} \Gamma(n+5 / 4) \Gamma(n+3 / 4)} d_{n}(\lambda), \quad n \geq 0,
$$

where

$$
\begin{aligned}
d_{n}(\lambda)= & (1-3 \lambda) \frac{\Gamma(n+1+\alpha / 2) \Gamma(n+1-\alpha / 2)}{\Gamma(1+\alpha / 2) \Gamma(1-\alpha / 2)} \\
& +\frac{3 \lambda \Gamma(n+3 / 2+\alpha / 2) \Gamma(n+3 / 2-\alpha / 2)}{\Gamma(3 / 2+\alpha / 2) \Gamma(3 / 2-\alpha / 2)}, \quad n \geq 0 .
\end{aligned}
$$

Then, $u$ is regular for every $\lambda \neq 0$ such that

$$
\lambda^{-1} \neq 3-\frac{\Gamma(n+3 / 2+\alpha / 2) \Gamma(n+3 / 2-\alpha / 2) \Gamma(1+\alpha / 2) \Gamma(1-\alpha / 2)}{\Gamma(n+1+\alpha / 2) \Gamma(n+1-\alpha / 2) \Gamma(3 / 2+\alpha / 2) \Gamma(3 / 2-\alpha / 2)}, \quad n \geq 0 .
$$

From (3.12) and (3.80)-(3.81), we obtain

$$
\tilde{a}_{n}=\frac{4 d_{n+1}(\lambda)}{(4 n+3)(4 n+5) d_{n}(\lambda)}, \quad n \geq 0 .
$$

Using (3.72), (3.83), and (3.16), we get

$$
\begin{aligned}
\gamma_{1} & =-\lambda, \\
\gamma_{2 n+2} & =\frac{4 d_{n+1}(\lambda)}{(4 n+3)(4 n+5) d_{n}(\lambda)}, \quad n \geq 0, \\
\gamma_{2 n+3} & =\frac{\left((2 n+2)^{2}-\alpha^{2}\right)\left((2 n+3)^{2}-\alpha^{2}\right) d_{n}(\lambda)}{4(4 n+5)(4 n+7) d_{n+1}(\lambda)}, \quad n \geq 0 .
\end{aligned}
$$

According to Proposition 3.13, the linear form $u$ is also Laguerre-Hahn. It satisfies (3.24) and (3.27) with

$$
\begin{gathered}
\Phi(x)=x^{2}\left(x^{2}-1\right), \quad B(x)=-\lambda^{-1} \frac{1-\alpha^{2}}{3} x^{4}, \quad \Psi(x)=-2\left(2-\lambda^{-1} \frac{1-\alpha^{2}}{3}\right) x^{3} \\
C_{0}(x)=-2 \lambda^{-1} \frac{1-\alpha^{2}}{3} x^{3}+2 x, \quad D_{0}(x)=\left(1-\lambda^{-1} \frac{1-\alpha^{2}}{3}\right) x^{2}+1-3 \lambda
\end{gathered}
$$

From (3.73), we have $-\tilde{\Phi}(0)-\lambda \widetilde{D}_{0}(0)=1-3 \lambda$ and $\Phi(0)=-1$.

Now it is enough to use Proposition 3.15 to obtain the following.

(i) If $\lambda$ satisfies (3.82) and $\lambda^{-1} \neq 3$, then the class of $u$ is $s=2$.

(ii) If $\lambda^{-1}=3$, then the class of $u$ is $s=1$. 
Again, from Proposition 3.16, we have for $n \geq 0$,

$$
\begin{gathered}
C_{0}(x)=-2 \lambda^{-1} \frac{1-\alpha^{2}}{3} x^{3}+2 x, \quad C_{1}(x)=2 x^{3}-6 \lambda x, \\
C_{2 n+2}(x)=4(n+1) x^{3}+\frac{6 \lambda \Gamma(n+3 / 2+\alpha / 2) \Gamma(n+3 / 2-\alpha / 2)}{d_{n}(\lambda)} x, \\
C_{2 n+3}(x)=2(2 n+3) x^{3}-\frac{6 \lambda \Gamma(n+5 / 2+\alpha / 2) \Gamma(n+5 / 2-\alpha / 2)}{d_{n+1}(\lambda)} x, \\
D_{0}(x)=\left(1-\frac{1-\alpha^{2}}{3 \lambda}\right) x^{2}+1-3 \lambda, \\
D_{2 n+1}(x)=(4 n+3) x^{2}, \\
=(4 n+5) \quad \\
\times\left(x^{2}-\frac{3 \lambda(1-3 \lambda) \Gamma(n+1+\alpha / 2) \Gamma(n+1-\alpha / 2) \Gamma(n+3 / 2+\alpha / 2) \Gamma(n+3 / 2-\alpha / 2)}{\Gamma(1+\alpha / 2) \Gamma(1-\alpha / 2) d_{n}(\lambda) d_{n+1}(\lambda)}\right) .
\end{gathered}
$$

(4) Let $v$ be the associated linear form of the first order of Bessel with parameter value of $\alpha=1$. We have $[8,11]$

$$
\begin{gathered}
\delta_{2 n+1}=\frac{-1}{(4 n+3)(4 n+5)}, \quad \delta_{2 n+2}=\frac{-1}{(4 n+5)(4 n+7)}, \quad n \geq 0, \\
\widetilde{\Phi}(x)=x^{2}, \quad \widetilde{\Psi}(x)=4 x, \quad \widetilde{B}(x)=-\frac{1}{3} \\
\widetilde{C}_{n}(x)=2(n+1) x, \quad \widetilde{D}_{n}(x)=2 n+3, \quad n \geq 0 .
\end{gathered}
$$

In this case, the linear form $v$ is a Laguerre-Hahn linear form of class $\widetilde{s}=0$.

By applying the same process as we did to obtain (3.57) and using the above results, we can get for $n \geq 0$,

$$
\widetilde{P}_{n}(0, \lambda)=\frac{\sqrt{\pi}}{2^{2 n+1} \Gamma(2 n+3 / 2)}(1-3 \lambda n(2 n+3)), \quad n \geq 0,
$$

then $u$ is regular for every $\lambda \neq 0$ such that

$$
\lambda^{-1} \neq 3 n(2 n+3), \quad n \geq 0 .
$$

From (3.12) and (3.89), we obtain

$$
\tilde{a}_{n}=-\frac{1-3 \lambda(n+1)(2 n+5)}{(4 n+3)(4 n+5)(1-3 \lambda n(2 n+3))}, \quad n \geq 0 .
$$


Using (3.87), (3.91), and (3.16), we get

$$
\begin{aligned}
\gamma_{1} & =-\lambda \\
\gamma_{2 n+2} & =-\frac{1-3 \lambda(n+1)(2 n+5)}{(4 n+3)(4 n+5)(1-3 \lambda n(2 n+3))}, \quad n \geq 0, \\
\gamma_{2 n+3} & =-\frac{1-3 \lambda n(2 n+3)}{(4 n+5)(4 n+7)(1-3 \lambda(n+1)(2 n+5))}, \quad n \geq 0 .
\end{aligned}
$$

By virtue of Proposition 3.13, the linear form $u$ is also Laguerre-Hahn. It satisfies (3.24) and (3.27) with

$$
\begin{gathered}
\Phi(x)=x^{4}, \quad B(x)=\frac{\lambda^{-1}}{3} x^{4}, \quad \Psi(x)=-2\left(2-\frac{\lambda^{-1}}{3}\right) x^{3}, \\
C_{0}(x)=\frac{2 \lambda^{-1}}{3} x^{3}, \quad D_{0}(x)=\left(1+\frac{\lambda^{-1}}{3}\right) x^{2}-3 \lambda .
\end{gathered}
$$

According to Proposition 3.15, the class of $u$ is $s=2$.

Finally, from Proposition 3.16, we have for $n \geq 0$,

$$
\begin{gathered}
C_{0}(x)=\frac{2 \lambda^{-1}}{3} x^{3}, \quad C_{1}(x)=2 x^{3}-6 \lambda x, \\
C_{2 n+2}(x)=4(n+1) x^{3}-\frac{6 \lambda}{1-3 \lambda n(2 n+3)} x, \\
C_{2 n+3}(x)=2(2 n+3) x^{3}+\frac{6 \lambda}{1-3 \lambda(n+1)(2 n+5)} x, \\
D_{0}(x)=\left(1+\frac{\lambda^{-1}}{3}\right) x^{2}-3 \lambda, \\
D_{2 n+1}(x)=(4 n+3) x^{2}, \\
D_{2 n+2}(x)=(4 n+5)\left(x^{2}-\frac{9 \lambda^{2}}{(1-3 \lambda(n+1)(2 n+5))(1-3 \lambda n(2 n+3))}\right) .
\end{gathered}
$$

\section{Acknowledgments}

We would sincerely like to express special thanks to the referees for their interest and never-failing support throughout the period of the project. Moreover, we are particularly indebted to them for their invaluable suggestions.

\section{References}

[1] J. Alaya and P. Maroni, Some semi-classical and Laguerre-Hahn forms defined by pseudo-functions, Methods and Applications of Analysis 3 (1996), no. 1, 12-30.

[2] _ Symmetric Laguerre-Hahn forms of class $s=1$, Integral Transforms and Special Functions 4 (1996), no. 4, 301-320.

[3] D. Beghdadi and P. Maroni, On the inverse problem of the product of a semi-classical form by a polynomial, Journal of Computational and Applied Mathematics 88 (1998), no. 2, 377-399.

[4] A. Branquinho and F. Marcellán, Generating new classes of orthogonal polynomials, International Journal of Mathematics and Mathematical Sciences 19 (1996), no. 4, 643-656. 
[5] T. S. Chihara, On co-recursive orthogonal polynomials, Proceedings of the American Mathematical Society 8 (1957), no. 5, 899-905.

[6] _ An Introduction to Orthogonal Polynomials, Gordon and Breach, New York, 1978.

[7] E. B. Christoffel, Über die Gaussische Quadratur und eine Verallgemeinerung derselben, Journal für die reine und angewandte Mathematik 55 (1858), 61-82.

[8] J. Dini, Sur les formes linéaires et les polynômes orthogonaux de Laguerre-Hahn, Thése, Universite Pierre et Marie Curie, Paris, 1988.

[9] J. Dini and P. Maroni, Sur la multiplication d'une forme semi-classique par un polynôme, Publ. Sem. Math. Univ. d'Antananarivo 3 (1989), 76-89.

[10] J. H. Lee and K. H. Kwon, Division problem of moment functionals, The Rocky Mountain Journal of Mathematics 32 (2002), no. 2, 739-758.

[11] F. Marcellán and E. Prianes, Perturbations of Laguerre-Hahn linear functionals, Journal of Computational and Applied Mathematics 105 (1999), no. 1-2, 109-128.

[12] P. Maroni, Sur la décomposition quadratique d'une suite de polynômes orthogonaux. I, Rivista di Matematica Pura ed Applicata (1990), no. 6, 19-53.

[13] Sur la suite de polynômes orthogonaux associée à la forme $u=\delta_{c}+\lambda(x-c)^{-1} L$, Periodica Mathematica Hungarica 21 (1990), no. 3, 223-248.

[14] Une théorie algébrique des polynômes orthogonaux. Application aux polynômes orthogonaux semi-classiques, Orthogonal Polynomials and Their Applications (Erice, 1990) (C. Brezinski, L. Gori, and A. Ronveaux, eds.), IMACS Ann. Comput. Appl. Math., vol. 9, Baltzer, Basel, 1991, pp. 95-130.

[15] - On a regular form defined by a pseudo-function, Numerical Algorithms 11 (1996), no. 1, 243-254.

[16] P. Maroni and I. Nicolau, On the inverse problem of the product of a form by a polynomial: the cubic case, Applied Numerical Mathematics 45 (2003), no. 4, 419-451.

M. Sghaier: Département de Mathématiques, Institut Supérieur des Sciences Appliquées et de Technologie de Gabès, Rue Omar Ibn El Khattab, 6072-Gabès, Tunisia

E-mail address: mabrouk.sghaier@issatgb.rnu.tn

J. Alaya: Faculté des Sciences de Gabès, Université de Gabès, Route de Mednine, 6029-Gabès, Tunisia

E-mail address: jilani.alaya@isggb.rnu.tn 


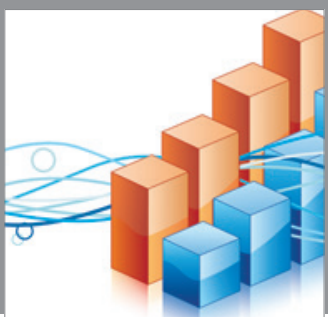

Advances in

Operations Research

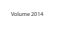

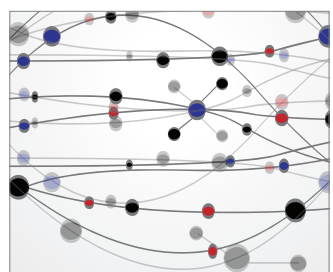

\section{The Scientific} World Journal
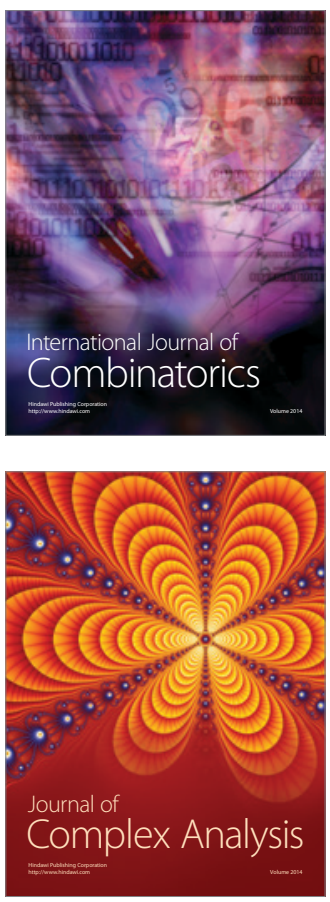

International Journal of

Mathematics and

Mathematical

Sciences
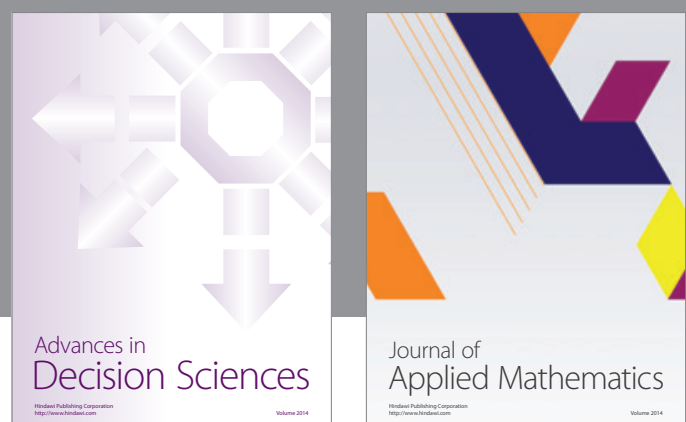

Journal of

Applied Mathematics
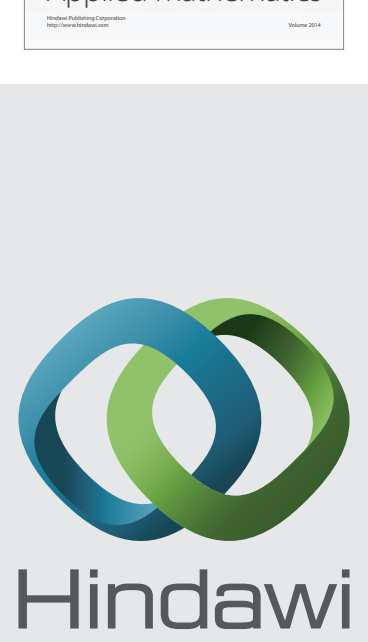

Submit your manuscripts at http://www.hindawi.com
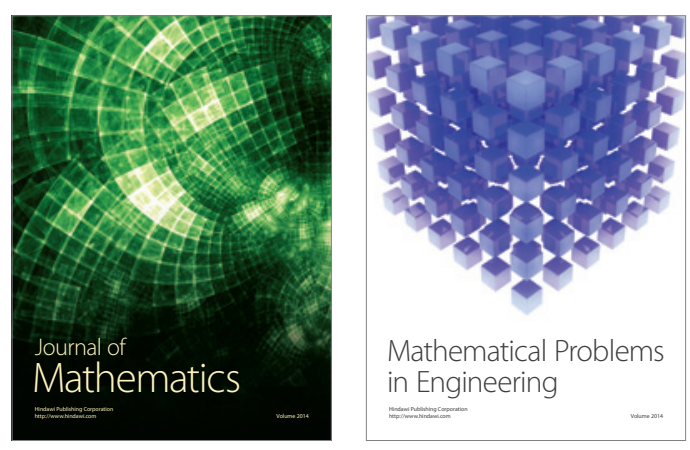

Mathematical Problems in Engineering
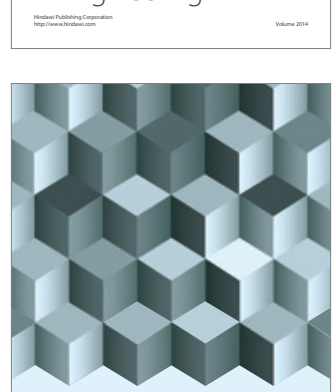

Journal of

Function Spaces
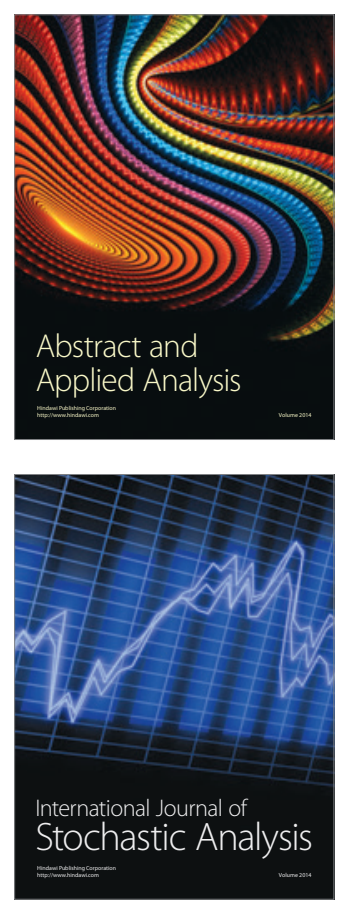

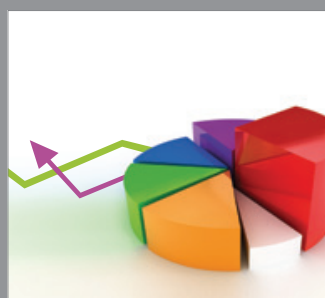

ournal of

Probability and Statistics

Promensencen
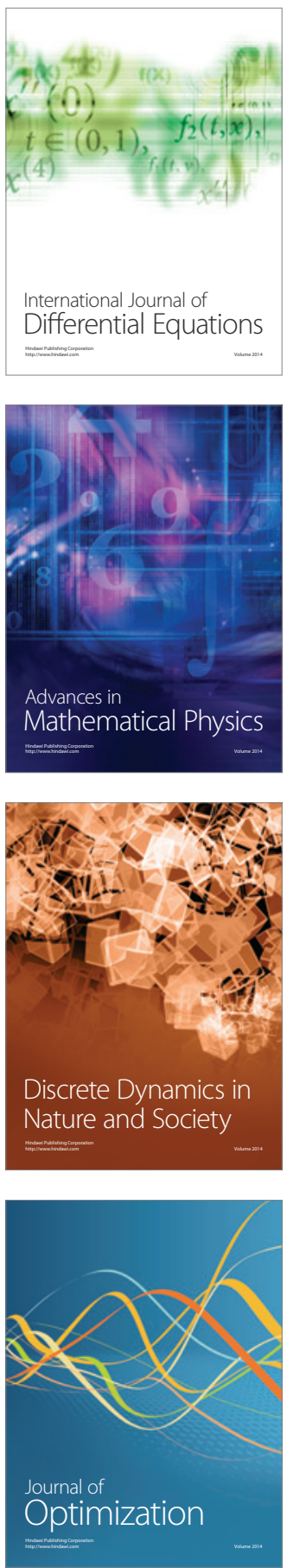